\title{
REDS: An Energy-Constrained Spatial Social Network Model
}

\author{
Alberto Antonioni ${ }^{1}$, Seth Bullock ${ }^{2}$, Marco Tomassini ${ }^{1}$ \\ ${ }^{1}$ Information Systems Department, Faculty of Business and Economics, University of Lausanne, Switzerland \\ ${ }^{2}$ Institute for Complex Systems Simulation, University of Southampton, United Kingdom
}

\begin{abstract}
The organisation of living systems is neither random nor regular, but tends to exhibit complex structure in the form of clustering and modularity. Here, we present a very simple model that generates random networks with spontaneous community structure reminiscent of living systems, particularly those involving social interaction. We extend the wellknown random geometric graph model, in which spatially embedded networks are constructed subject to a constraint on edge length, in order to capture two key additional features of organic social networks. First, relationships that span longer distances are more costly to maintain. Conversely, relationships between nodes that share neighbours may be less costly to maintain due to social synergy. The resulting networks have several properties in common with those of organic social networks. We demonstrate that the model generates nontrivial community structure and that, unlike for random geometric graphs, densely connected communities do not simply arise as a consequence of an initial locational advantage.
\end{abstract}

\section{Introduction}

The structure of living systems is neither random (where every system element interacts with a random sub-set of other elements) nor regular (where elements interact with neighbours on a lattice). Instead, such systems tend to exhibit complex structure typically featuring clustering and modularity. No doubt much of the detail of this structure arises for reasons that are specific and idiosyncratic to each case. However, self-organisation in simple systems suggests that some characteristic structure may be relatively generic and may arise as a result of fairly simple factors-indeed this type of self-structuring may serve as an important foundation for subsequent evolution and development (e.g., Boerlijst and Hogeweg, 1991; Di Paolo, 2000).

Here we pursue this idea in the context of a social network model (see Toivonen et al., 2009, for an overview of such models). We demonstrate that simple constraints on random network formation due to spatial embedding, limited energy, and the influence of social synergy can generate structures that exhibit key features of social networks: high clustering, right-skewed degree distribution, positive degree assortativity, and strong community structure.
The article is organized as follows. In the next section we give a brief introduction to random geometric graphs, including an energy constrained variant of this network class. Subsequently, we present the REDS model, an extension to the energy-constrained random geometric graph that allows for social synergy to mitigate the costs of maintaining inter-node relationships. The following section presents and discusses the main numerical results, demonstrating that the new model is capable of generating networks that share key properties with real-world social networks. The paper concludes with a summary of the key findings.

\section{Social and Spatial Network Models}

Most studies of complex networks, including social networks, consider relational networks where physical distances between nodes are not represented. However, most systems, including social, biological and environmental networks, are embedded in Euclidean space (see Barthélemy, 2011, for a recent review of the field). While relational social network models are important and have been studied in depth (see, e.g., Vázquez, 2003; Catanzaro et al., 2004; Toivonen et al., 2006; Kumpula et al., 2007), their spatial aspects are less well explored (but see Boguñá et al., 2004; Wong et al., 2006; Serrano et al., 2008; zu ErbachSchoenberg et al., 2014, for some recent attempts).

The canonical spatial network model is the Random Geometric Graph (RGG). We shall use this simple model as the foundation for the work presented here. A RGG is obtained when points located in a plane are connected according to a geometric rule, e.g., connect all pairs of nodes separated by less than a threshold distance, $R$. There is an extensive mathematical literature on random geometric graphs, particularly in the context of continuum percolation (Dall and Christensen, 2002; Penrose, 2003; Barthélemy, 2011).

In order to generate an $N$-node RGG with distance threshold, $R$, distribute $N$ nodes uniformly at random in the unit space, $\Omega \in \mathbb{R}^{2}$, and add an edge between every pair of nodes separated by a distance $r<R$, using the standard Euclidean metric on $\mathbb{R}^{2}$. Furthermore, unless otherwise noted, here we shall assume that the unit space, $\Omega$, is the square $[0,1]^{2}$ with 
cyclic boundary conditions (i.e., a torus).

Several RGG variants exist. For example, the Manhattan distance is sometimes used to model mobility networks (Glauche et al., 2003). The general properties of these networks are very close to those employing the more common Euclidean distance, which are the ones we describe here.

The average degree, $\bar{k}$, of a RGG can be easily estimated as $\bar{k}=\rho V$, where $\rho=N$ is the node density, i.e., the number of nodes per unit space, and $V$ is the neighborhood area. In this case $\bar{k}=N \pi R^{2}$. The degree distribution of RGGs with a sufficiently large number of nodes can be estimated by the Poisson distribution with parameter $\lambda=\bar{k}$ (Dall and Christensen, 2002).

For large $N$, the clustering coefficient of a RGG (i.e., the average over all individual node's clustering coefficients, Newman, 2010) tends to $1-\frac{3 \sqrt{3}}{4 \pi} \sim 0.5865$ for all 2dimensional RGGs in the Euclidean space (Dall and Christensen, 2002). This important result depends on the particular construction of RGGs. The average clustering coefficient tends to the ratio of the average shared neighborhood area of two connected nodes to the whole neighborhood area. It is clear that changing the radius, $R$, does not alter this value.

RGGs exhibit positive assortativity, i.e., there is a positive correlation between the degree of pairs of connected nodes (Boccaletti et al., 2006). Antonioni and Tomassini (2012) demonstrate that the assortativity of any $d$-dimensional RGG tends to the value of its average clustering coefficient (a similar result was presented by Barnett et al., 2007, for spatial networks more generally). Many more properties of RGGs are derived by Penrose (2003).

Energy constrained RGGs (Antonioni et al., 2013, hereafter EC-RGGs) are an extension to the standard RGG model where each of a node's connections costs an amount of energy equivalent to its Euclidean length. In addition to the standard constraint that each edge cannot cost more than $R$, the total cost of an individual node's edges may not exceed some finite threshold value, E. Networks are constructed by assigning legal edges at random until no more edges can be afforded. For large E, EC-RGGs tend to become equivalent to RGGs, saturating such that all edges of length less than $R$ are present in the graph. Where both $E$ and $R$ are large, complete graphs are obtained. However, where both $E$ and $R$ are limiting factors, EC-RGG graphs exhibit a range of clustering and (positive) assortativity values (unlike RGGs). However, neither RGGs nor EC-RGGs exhibit the skewed degree distributions and community structure that are characteristic of social networks.

\section{The REDS Model}

The REDS model builds on the RGG and EC-RGG models by including and parameterising the positive influence of shared network neighbours on the cost of maintaining relationships (see Fig. 1). The intuitions here are that (i) there is a limit to the distance over which a relationship can be

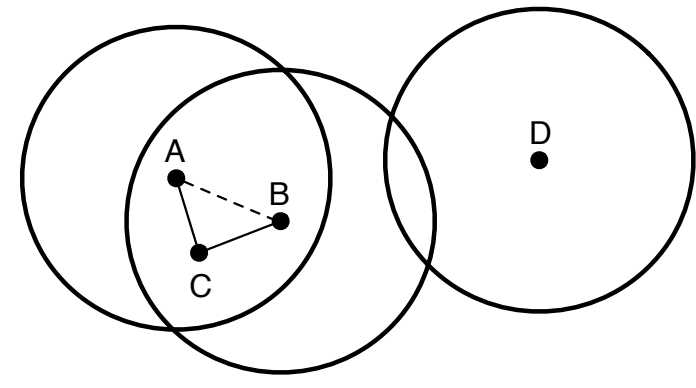

Figure 1: Nodes (dots) may become linked by edges (solid lines) only if (i) they fall within each other's social reach (circles), excluding the possibility of edges $A D$ and $B D$, and (ii) they can afford to. The cost of edge $A B$ increases with the distance between $A$ and $B$, but may be reduced by the presence of any shared neighbours of $A$ and $B$, e.g., $C$.

maintained, (ii) relationships between nodes vary in terms of cost, (iii) longer distance relationships cost proportionately more than shorter distance relationships, and (iv) relationships with individuals that are themselves connected together may be cheaper to maintain.

This last intuition is exemplified as follows. If I have two friends that know each other, in order to keep my relationship with each of them alive I don't have to physically visit or interact with each of them to the same extent that I would have to in order to maintain two unconnected friends. Direct interaction with one friend effectively involves an element of indirect interaction with friends that we share in common through gossip, chance encounters, group gatherings, etc. In more general terms, this is a local network effect that represents the potential for synergetic or catalytic interactions between the system elements.

The REDS model thus comprises four components:

1. Reach: an undirected edge, $i j$, between a pair of nodes, $i$ and $j$, may only exist if the Euclidean distance between them, $D_{i j}$, is less than their "social reach", $R$.

2. Energy: each node, $i$, has a finite quantity of "social energy", $E$, that may be spent on maintaining its edges.

3. Distance: the cost, $c_{i j}$, of edge $i j$ is proportionate to the Euclidean "social distance", $D_{i j}$, between $i$ and $j$.

4. Synergy: the cost, $c_{i j}$, of edge $i j$ varies inversely with the number of network neighbours that $i$ and $j$ share, $k_{i j}$. This effect is parameterised using $0 \leq S \leq 1$.

More explicitly, the cost of each edge is calculated as:

$$
c_{i j}=\frac{D_{i j}}{1+S k_{i j}},
$$


where $k_{i j}$, the number of neighbours shared by $i$ and $j$, is the cardinality of the intersection between the set of $i$ 's neighbours and the set of $j$ 's neighbours.

Thus, when $S=0$ the model reduces to the energy constrained RGG model, with $c_{i j}=D_{i j}$. However, where $0<S \leq 1$, the model incorporates a local network effect that reduces the cost of edges between nodes that have network neighbours in common. Where $S=0$ each relationship must be maintained independently, whereas for positive $S$, while maintaining each relationship always involves a non-zero cost, these costs are lower for relationships that involve nodes with shared neighbours. For example, when two friends meet they may discuss or interact with common friends, reinforcing those relationships at a cost less than that of visiting or interacting with all neighbours individually.

The construction process to build an $N$-node REDS network with social reach $R$, social energy $E$, and social synergy $S$ can be summarized as follows:

1. A population of $N$ nodes are distributed uniformly at random in the unit square $\Omega \in \mathbb{R}^{2}$. Each node, $i$, is allocated the same initial energy, $E_{i}=E$.

2. A node $i$ is picked uniformly at random from the population, and a second node $j$ is chosen uniformly at random from the set of nodes for which the Euclidean distance $D_{i j}<R$.

3. An undirected edge between $i$ and $j$ is created only if both nodes have sufficient energy to afford the new set of neighbours that would result. For $i$, this condition is met if $E_{i} \geq \sum_{x} c_{i x}^{+i j}$, where $c^{+i j}$ denotes the cost of an edge in the updated graph including the new edge $i j$, and $x$ are the neighbours of $i$ in this updated graph. The same condition must hold for $j$, mutatis mutandis.

4. Steps 2 and 3 are repeated until no more edges can be created according to the linking rule.

The maximum cost that a node, $i$, may need to pay in order to maintain its $k$ edges occurs when either $S=0$ (no synergy) or none of $i$ 's neighbours are connected to each other, i.e., for each neighbour, $j$, of $i, k_{i j}=0$. In such a case, the total cost to $i$ is $\sum_{j} D_{i j}$ which is the sum of all the distances from $i$ to its neighbours. This is appropriate, since the worst case scenario is that a node must pay to maintain each of its relationships independently.

The minimum cost that a node, $i$, may need to pay in order to maintain its $k$ neighbours occurs for scenarios where $S=1$ and where $i$ 's neighbours form a perfect clique, i.e., for each neighbour, $j$, of $i, k_{i j}=k-1$. In such a case, the total cost to $i$ is $\sum_{j} \frac{D_{i j}}{1+k-1}=\frac{1}{k} \sum_{j} D_{i j}$ which is the average distance from $i$ to one of its $k$ neighbours. This is appropriate, since perfect synergy (maximum $S$ ) should not reduce the cost of a set of neighbours to less than the cost of maintaining a relationship with one of them.
Notice that edges are undirected and edge costs are symmetric, with $c_{i j}=c_{j i}$. However, it may be the case that while $i$ can afford a potential new edge, $i j$, the same edge is not affordable for $j$ as a result of $i$ and $j$ having differing existing edge costs that result in $j$ not having enough remaining energy. Such an edge would not be added to the network, since both of the nodes must be able to afford a new edge connecting them, Notice also that (for $S>0$ ) as edges are added to a network, the cost of both existing network edges and potential new edges may change as a consequence of the creation of new shared neighbours. Thus, even if the number of edges in a graph increases monotonically during construction, the amount of residual energy available to individual nodes (and to the network itself) may sometimes increase (although no node ever has access to more than its initial allocation of energy $E_{i}$ ). Thus, unlike both RGGs and EC-RGGs. the construction of a REDS network may be path dependent.

\section{Results}

Figure 2 shows three example networks generated by the REDS model: no synergy (top), high synergy (middle), and no synergy compensated for with increased energy (bottom). All three graphs share the same value of $N=10^{3}$ and $R=0.1$, and all exhibit some clustering (the presence of triangle motifs in the network), positive assortativity (highdegree nodes tend to be directly connected to other highdegree nodes at a greater than chance frequency) and community structure (sets of nodes exist, within which pairs of nodes are more likely to be connected to each other than to nodes outside the set).

The no-synergy network (Fig. 2-Top) is sparsely connected and lacks distinctive community structure, whereas a network of equivalent energy but increased social synergy (Fig. 2-Middle) has (i) increased mean degree, because social synergy ensures that some edges become cheaper to maintain, and (ii) stronger community structure, because social synergy ensures that adding edges during network construction tends to reduce the cost of nearby edges involving the same nodes, rather than edges in general. A network with no social synergy but increased energy (Fig. 2-Bottom) is capable of achieving the same mean degree as the highsynergy network, but does not achieve its high clustering, assortativity and heterogeneous community structure.

Figure 3 presents a more comprehensive picture of how the mean degree, mean clustering and assortativity of REDS networks vary with model parameters. Each heat map evidences two sharply defined regimes in the $S \times E$ plane of the model's parameter space": the "saturated" regime and the "sparse" regime.

\footnotetext{
${ }^{1}$ We hold $N$ and $R$ constant since they can be thought of as defining a "scale" for the model in terms of node density, $N$, and the average distance between potential neighbours, $2 R / 3$.
} 

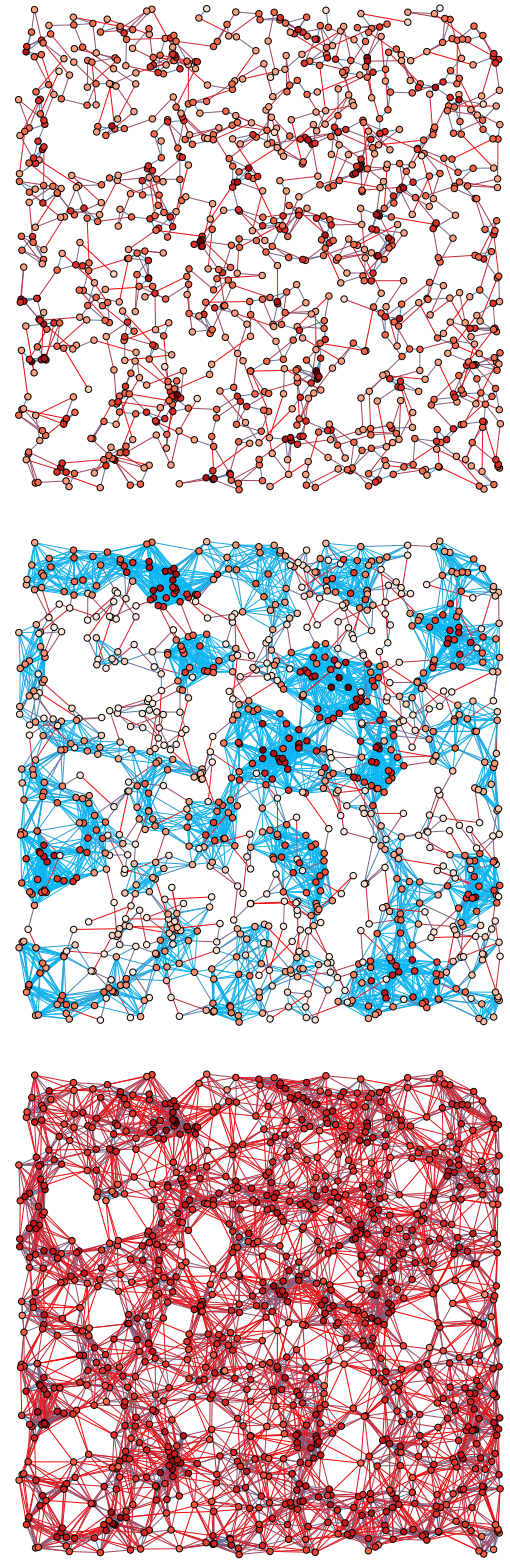

Figure 2: Example REDS networks $\left(N=10^{3}, R=0.1\right)$. Red nodes have higher degree; red edges have higher cost and blue edges lower cost. Top $(S=0, E=0.15)$ : no synergy results in a sparse $(\bar{k}=3)$ graph with modest clustering (0.1) and assortativity (0.33). Middle $(S=1, E=0.15)$ : maximum synergy results in a dense graph $(\bar{k}=12)$ with stronger clustering (0.5) and assortativity (0.65), and evident community structure. Bottom $(S=0, E=0.9)$ : no synergy, but sufficient energy to match the middle graph's density $(\bar{k}=13.7)$, results in lower clustering (0.34) and assortativity (0.16) and less evident community structure. (Nb. For illustrative clarity, networks were constructed on a bounded unit square, here, rather than a torus.)

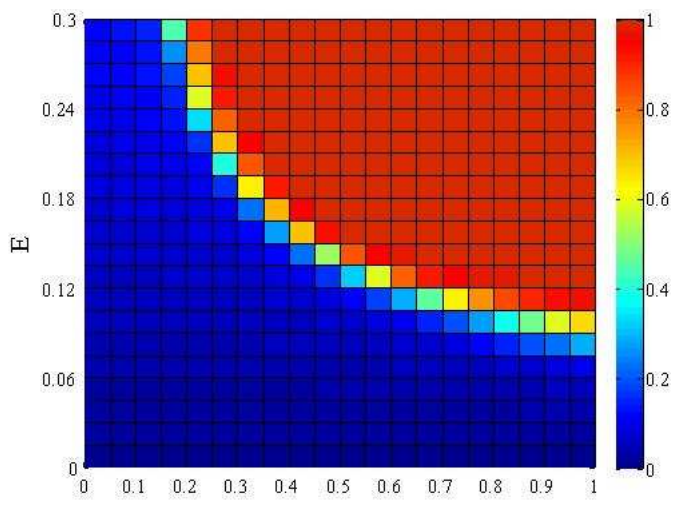

$\mathrm{S}$

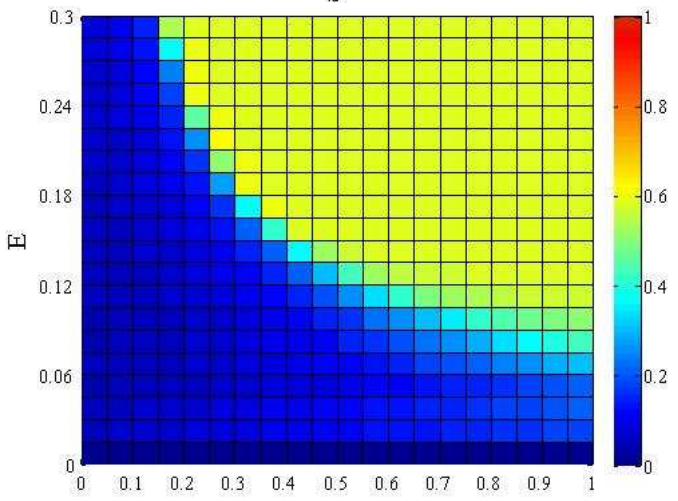

$\mathrm{S}$

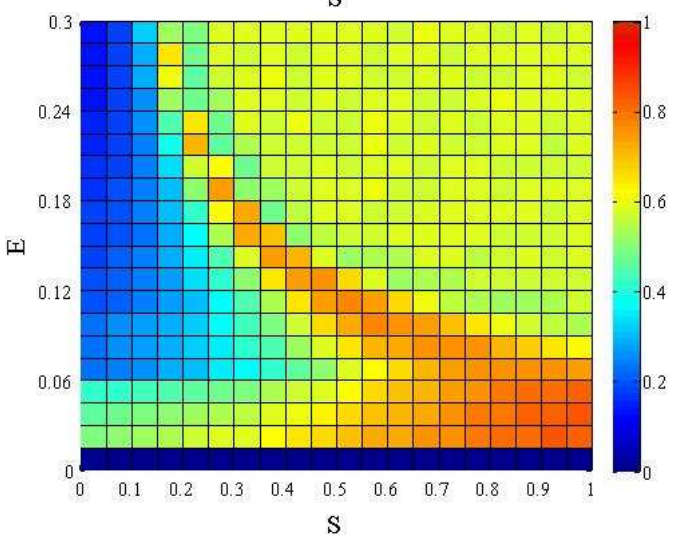

Figure 3: Mean degree (top), clustering coefficient (middle) and assortativity (bottom) for REDS networks $\left(N=10^{4}\right.$, $R=0.05$ ). Each cell averages 10 independent networks. Mean degree is normalized w.r.t. $k_{\max }=N \pi R^{2}(\sim 78.54$, the mean degree for a RGG where $N=10^{4}$ and $R=0.05$ ). Clustering is calculated as the mean node clustering coefficient. For large $E$ and/or $S$, a distinct "saturated" regime exists where degree $\sim 78.54$, clustering $\sim 0.5865$ and assortativity also $\sim 0.5865$ (i.e., the values predicted for RGGs of equivalent density by Dall and Christensen, 2002; Antonioni and Tomassini, 2012). Outside this regime, degree and clustering are somewhat lower, while assortativity varies considerably with $S$ and $E$. (For $E=0$, the empty graph obtains.) 
The saturated regime is characterised by high $E$ and high $S$ and is associated with REDS networks that are equivalent to saturated RGGs. This regime corresponds to scenarios in which each node starts with enough energy to accumulate connections to all of the $k=N \pi R^{2}$ nodes that lie within its social reach. This threshold value for $E$ is high when $S=0$, because each edge must be paid for independently, but it decreases rapidly as $S$ increases, since synergy reduces the energy that must be spent on edges that close triangles.

Where synergy is minimal $(S=0)$, the total cost incurred by a node, $i$, when connecting to all of the $k$ nodes within its social reach is $\sum D_{i j} \forall j: D_{i j}<R$. Since the mean distance to a neighbour is just $2 R / 3$, the boundary between the two regimes is $E=2 N \pi R^{3} / 3$ for $S=0(\sim 2.61$ for the scenarios plotted in Fig. 3). Where synergy is maximal $(S=1)$, each node still requires some non-zero amount of energy in order to connect to all of the nodes within its social reach. In such a scenario, if a node, $i$, were able to form a perfect clique with all of the nodes within its social reach, the total cost of $i$ 's edges would be equal to $\frac{1}{k} \sum D_{i j}$ $\forall j: D_{i j}<R$, i.e., the average distance to a node within its social reach, or $2 R / 3(0.03 \dot{3}$ for the cases plotted in Fig. 3$)$.

However, this value is a lower bound that cannot be reached in practice. First, during the network construction process, before a node can come to be part of minimally expensive clique it must first be part of an incomplete clique that is necessarily more expensive. Therefore, nodes must have access to more energy than is required by the lower bound calculation considered above. It is also the case that the order in which nodes accumulate edges will tend to impact on the extent to which they can achieve a maximal final degree. Second, spatial constraints ensure that a node cannot form a perfect clique with every node within its social reach since some of these nodes will be separated by a distance greater than $R$ and therefore cannot themselves become neighbours. Consequently, for networks where $S=1$, the regime boundary will tend to occur at $E>2 R / 3$.

The saturated regime transitions sharply to a "sparse" regime within which REDS networks are very different from RGG networks. For very low values of $E$ and/or $S$ these networks become fragmented, with nodes unable to afford to maintain more than a few neighbours. However, with moderate values of $S$ and $E$ (below the regime threshold), we find networks that, although sparsely connected by comparison with RGGs, still exhibit significant clustering and a wide range of positive assortativity values, including values that are significantly higher than those of RGGs. This high assortativity indicates the presence of dense pockets of highdegree nodes separated by a hinterland of low-degree nodes connected together (e.g., Fig. 2-Middle).

Moreover, these sparse-regime networks exhibit degree distributions that are very different from the Poisson distributions characteristic of RGGs in the saturated regime (see Fig. 4). Sparse-regime networks tend to exhibit degree distributions that are more sharply peaked than a Poisson and (for some values of $S$ and $E$ ) significantly more positively skewed. Interestingly, at and around the boundary between the two regimes, we see degree distributions that are a super-position of the individual distributions associated with each regime, suggesting that while some parts of the network have managed to achieve RGG-like configuration, others have not been able to do so.

More generally, it is instructive to ask: to what extent does a node's final status within the network depend on its initial location in the spatial distribution? Although the distribution of nodes is uniform random, there will necessarily be some variation in the local conditions that each node experiences. Some nodes will have access to more or less potential neighbours within their social reach, $R$. It might be expected that nodes that are initially disadvantaged by being located in a more sparsely populated patch of space could tend to end up with fewer neighbours in the final graph. Might this effect be responsible for the patches of densely interconnected nodes separated by "hinterland" regions of relatively sparsely connected nodes in some networks?

Figure 5 goes some way towards answering this question by plotting, for the same range of REDS networks displayed in Fig. 4, the final degree achieved by network nodes against the maximum degree that the nodes would have achieved had they been able to connect to every node within their social reach. This figure again reflects the two regimes that we have seen in previous figures. In the saturated regime, the RGG-like networks necessarily exhibit a strong identity relationship between the degree that a node achieves and the maximum degree that it could achieve given the availability of potential neighbours within its reach.

However, for networks within the sparse regime, the relationship between potential degree and actual realised degree is very different. Node degree here is obviously lower in general, but it is also not predicted by the number of potential neighbours within reach. Whether a node is advantaged or disadvantaged by the number of neighbours available at the location in which they are placed has little to do with the degree that they ultimately attain. Indeed, for moderate $E$ and $S$ within this regime (where degree distributions are wider due to the availability of energy and the relative cheapness of some triangle-closing edges) having an average starting location might be most beneficial (e.g., $S=0.5$, $E=0.09$ ). Again, as with previous figures, we see an interesting hybrid effect at the regime boundary. For instance, where $S=0.75$ and $E=0.09$, a large sub-population of nodes exhibit a (slightly depressed) RGG-like distribution, while the remaining nodes are distributed as per a regular sparse regime network. This effect is even more pronounced for $S=1$ and $E=0.09$. Again, such scatterplots can be interpreted in terms of hybrid networks within which some spatial regions are close to achieving RGG-like configurations, but other regions are not. 


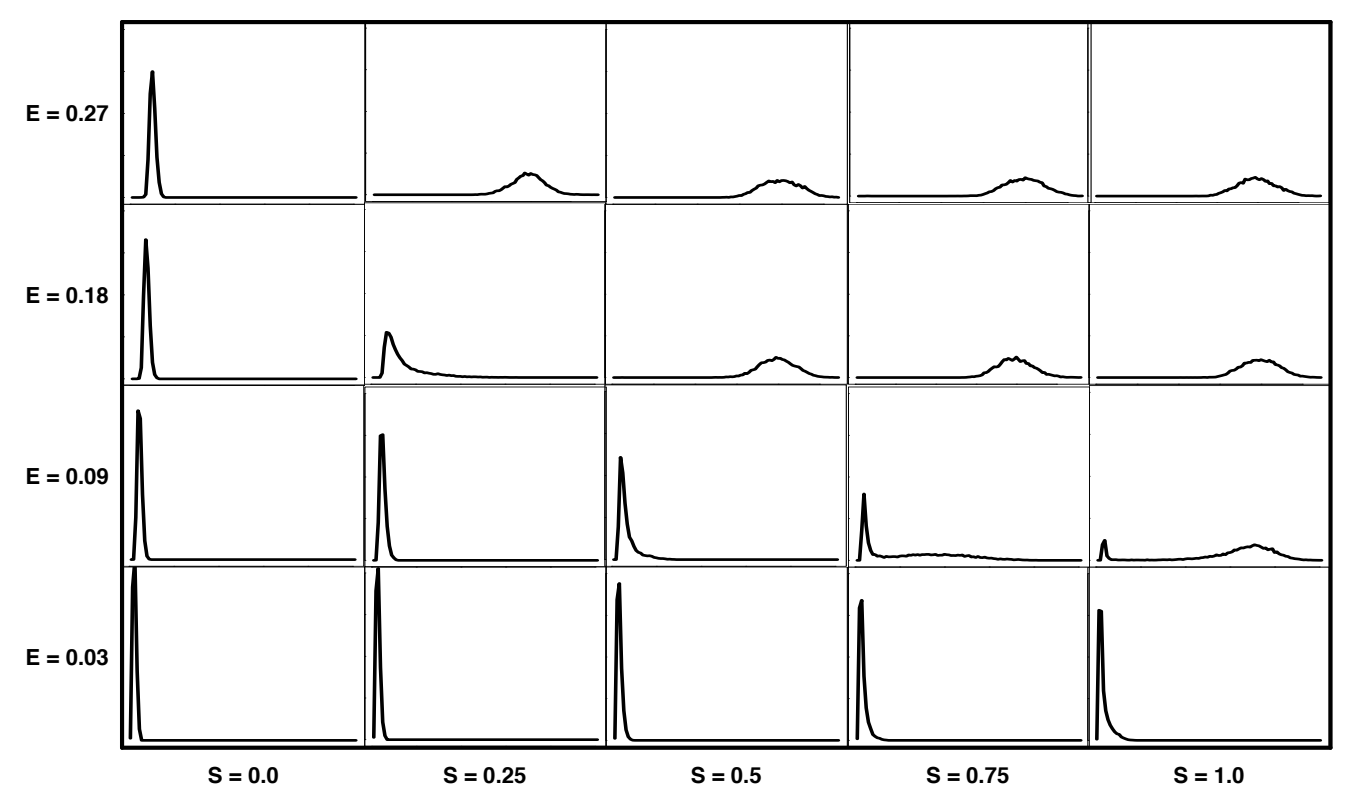

Figure 4: Degree distributions for REDS networks $\left(N=10^{4}, R=0.05\right)$. Sub-plot $x$-axes represent node degree: $0 \leq k \leq 100$; $y$-axes represent the number of nodes with that degree: $0 \leq p(k) \leq 4000$. Two distinctive regimes exist: the "saturated" distribution is Poisson with $\lambda=\bar{k}_{\max } \sim 78.54$; the "sparse" distribution is sharply peaked and can be positively skewed. At the boundary between the regimes, hybrid multi-modal distributions can be observed, e.g., for $S=1.0, E=0.09$.

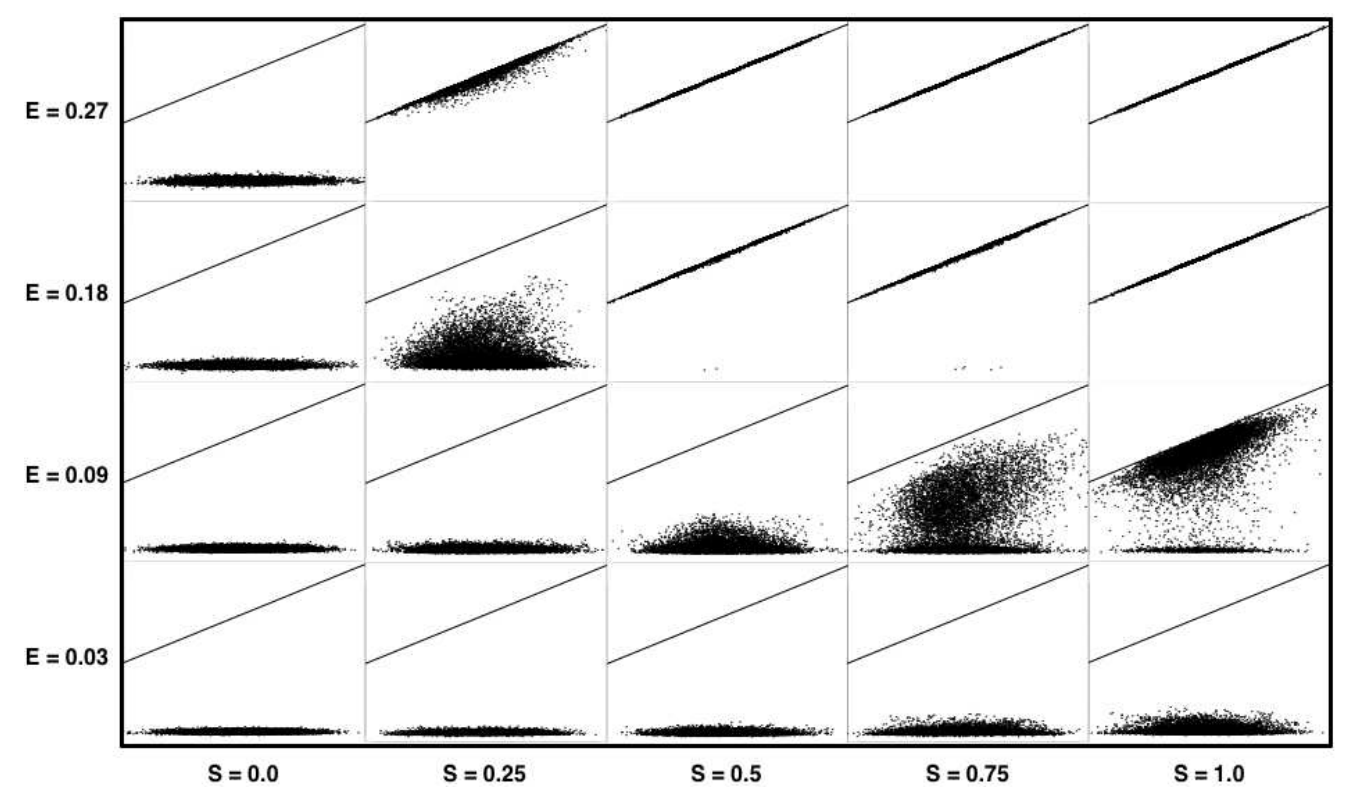

Figure 5: Scatterplots of realised degree (y) against maximum potential degree $(x)$ for REDS networks $\left(N=10^{4}, R=0.05\right)$. Sub-plot $x$-axes represent maximum potential node degree: $50 \leq n \leq 110 ; y$-axes represent actual realised node degree: $0 \leq k \leq 110$. (A small quantity of jitter noise $(<5 \%)$ has been added to better indicate density where many datapoints have identical locations.) Again, two distinctive regimes exist: the "saturated" distribution ranges along the line $y=x$ (the upper bound on node degree) with maximum degree predicting realised degree; the "sparse" distribution shows little effect of potential degree on realised degree. Again, hybrid distributions can be observed at the boundary between the regimes, e.g., for $S=1.0, E=0.09$. 

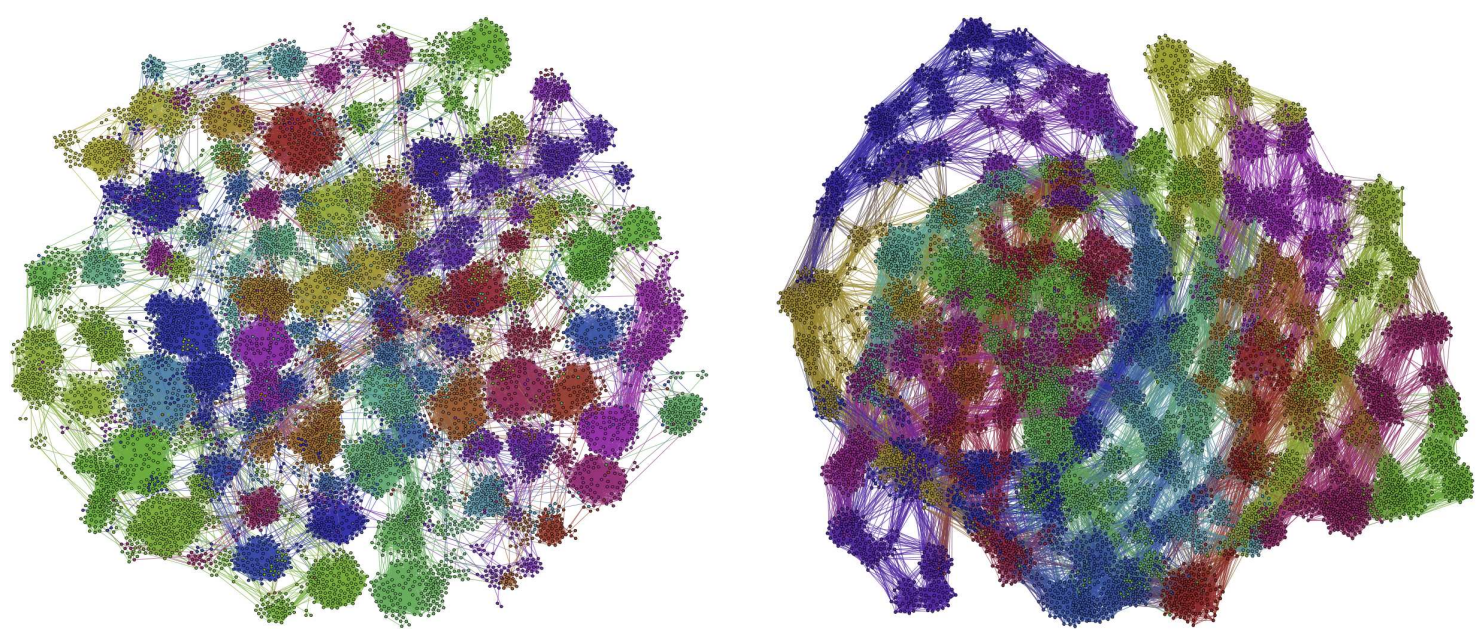

Figure 6: Community structure projections for two REDS networks with differing synergy but similar degree $\left(N=10^{4}\right.$, $R=0.05, \bar{k} \sim 13.5)$. Left $(S=1.0, E=0.065)$ : High synergy results in many (49) sharply defined communities (average clustering coefficient $=0.38$, modularity $=0.95)$. Right $(S=0.1, E=0.3)$ : Low synergy results in fewer (24) lesswell defined communities (average clustering coefficient $=0.2$, modularity $=0.865$ ). Nodes are coloured by community membership. Each layout relocates nodes to reflect their network relationships rather than their original co-ordinates. The OpenOrd algorithm was used for visual representation (Martin et al., 2011). Modularity was calculated using the fast unfolding algorithm due to Blondel et al. (2008). (Nb. For illustrative clarity, networks were constructed on a bounded unit square, here, rather than a torus.)

Figure 6 depicts two REDS networks non-spatially in order to reveal the community structure that they exhibit. Each network is projected onto the 2- $d$ plane in such a way as to reflect its relational, rather than spatial, organisation. Modularity analysis was carried out using the algorithm due to Blondel et al. (2008). By colouring the nodes according to which community they are assigned, we can get a sense of the effect of social synergy on community structure. Whereas the high-synergy network presents a large number of distinct and well-separated communities, the low-synergy network (despite having the same average degree and same spatial distribution of nodes) presents less community structure with fewer resolved communities and more interaction between them. This is consistent with the results presented above, since in the absence of social synergy the uniform distribution of nodes tends to generate an undifferentiated blanket of connectivity, whereas in the presence of social synergy genuine community structure arises and organises in a way that is constrained by distance and energy, but tends to transcend the original spatial layout of the nodes.

\section{Discussion}

In the previous section we were able to demonstrate that the number of potential neighbours within a node's reach was not a predictor of its eventual degree for networks within the sparse regime. However, we did not demonstrate what property or properties of a node did predict this outcome of the network construction process.
It is likely (although yet to be confirmed) that, in the sparse regime where $S>0$, whether a node achieves a high or low degree during network construction is determined by the first few edges that are allocated in its locale, rather than the number of potential neighbours within its reach. Nodes that are lucky enough to be assigned edges early in the construction process will enjoy the same kind of rich-get-richer advantage that is enjoyed by nodes that arrive early during a process of preferential attachment (Barabási and Albert, 1999). While preferential attachment explicitly biases network growth in favour of well-connected nodes through its global choice mechanism, the current model achieves something similar by encouraging clique-ish sub-graphs to form around focal nodes with higher than average local clustering.

It is also likely (although yet to be confirmed) that nodes that connect initially to relatively near-by neighbours (rather than other affordable neighbours that are more distant but still within reach) are advantaged during the construction process. Expending energy on connecting with a relatively near-by node means more energy remains to be spent on a new neighbour, and also increases the chance that such a new neighbour can (afford to) close a triangle with you and your first near-by neighbour.

In order to explore this issue, and to better characterise the saturated/sparse regime boundary, it may be useful to consider a "greedy" version of the model in which, rather than picking a random affordable edge, nodes select the cheapest possible new edge. 


\section{Conclusion}

In this article we have proposed an original model for the construction of social networks that are spatially embedded, constrained by limited energy, and influenced by some degree of social synergy. We started from the random geometric graph model and added three additional ingredients in order to generate networks that possess several of the statistical features exhibited by actual spatial social networks.

The main idea is to attribute a limited but equal amount of social energy to each of a set of spatially embedded nodes. Nodes can spend this resource to link to other nodes as a function of their Euclidean distance, longer links being more expensive than shorter ones, but this cost may be offset by the catalytic or synergetic effect of shared social connections. In this way we obtain networks that resemble realworld networks from the point of view of their statistical features. In particular, the generated networks have high clustering, positive degree correlation, and the presence of community structure. Within a "saturated" regime the model recovers the properties of random geometric graphs, but outside this regime there exists an interesting and varied class of networks that may exhibit degree distributions and community structure reminiscent of organic modular networks.

The model presents several possibilities for further research with the purpose of understanding more about the generic properties of networks inspired by constraints on social processes. For example, one could assume that nodes are not static in space, but move from place to place, perhaps stretching or breaking connections as they do so. This type of process may have the potential to introduce the kind of long-distance links that reduce the characteristic path length of small world networks. Furthermore, it might be reasonable to consider heterogeneity in the distribution of social energy or social reach or social synergy among the nodes. The linking process is bilateral in the present version, i.e., both partners must pay the same amount of energy to create the connection. One-way links could also be considered and the model could be extended to make it dynamical allowing for link removal as well as link formation.

Acknowledgments: We thank the referees for their comments. Bullock was supported by EPSRC grant EP/H021698/1.

\section{References}

Antonioni, A., Egloff, M., and Tomassini, M. (2013). An energybased model for spatial social networks. In Liò, P., Miglino, O., Nicosia, G., Nolfi, S., and Pavone, M., editors, Advances in Artificial Life, ECAL 2013, pages 192-199. MIT Press.

Antonioni, A. and Tomassini, M. (2012). Degree correlations in random geometric graphs. Physical Review E, 86:037101.

Barabási, A.-L. and Albert, R. (1999). Emergence of scaling in random networks. Science, 286:509-512.

Barnett, L., Di Paolo, E., and Bullock, S. (2007). Spatially embedded random networks. Physical Review E, 76(5):056115.
Barthélemy (2011). Spatial networks. Physics Reports, 499:1-101.

Blondel, V. D., Guillaume, J.-L., Lambiotte, R., and Lefebvre, E. (2008). Fast unfolding of communities in large networks. Journal of Statistical Mechanics, 2008:P10008.

Boccaletti, S., Latora, V., Moreno, Y., Chavez, M., and Hwang, D.-U. (2006). Complex networks: Structure and dynamics. Physics Reports, 424:175-308.

Boerlijst, M. C. and Hogeweg, P. (1991). Spiral wave structures in pre-biotic evolution: Hypercycles stable against parasites. Physica D, 48:17-28.

Boguñá, M., Pastor-Satorras, R., Díaz-Guilera, A., and Arenas, A. (2004). Models of social networks based on social distance attachment. Physical Review E, 70:056122.

Catanzaro, M., Caldarelli, G., and Pietronero, L. (2004). Assortative model for social networks. Physical Review E, 70(3):037101.

Dall, J. and Christensen, M. (2002). Random geometric graphs. Physical Review E, 66:016121.

Di Paolo, E. A. (2000). Ecological symmetry breaking can favour the evolution of altruism in an action-response game. Journal of Theoretical Biology, 203:135-152.

Glauche, I., Krause, W., Sollacher, R., and Greiner, M. (2003). Continuum percolation of wireless ad hoc communication networks. Physica A, 325:577-600.

Kumpula, J. M., Onnela, J.-P., Saramäki, J., Kaski, K., and Kertész, J. (2007). Emergence of communities in weighted networks. Physical Review Letters, 99:228701.

Martin, S., Brown, W. M., Klavans, R., and Boyack, K. W. (2011). Openord: An open-source toolbox for large graph layout. In Proc. SPIE 7868, Visualization and Data Analysis 2011. 786806.

Newman, M. E. J. (2010). Networks: An Introduction. Oxford University Press, Oxford, UK.

Penrose, M. (2003). Random Geometric Graphs. Oxford University Press, Oxford, UK.

Serrano, M., Krioukov, D., and Boguná, M. (2008). Self-similarity of complex networks and hidden metric spaces. Physical Review Letters, 100:078701.

Toivonen, R., Kovanen, L., Kivelä, M., Onnela, J.-P., Saramäki, J., and Kaski, K. (2009). A comparative study of social network models: Network evolution models and nodal attribute models. Social Networks, 31:240-254.

Toivonen, R., Onnela, J.-P., Saramäki, J., Hyvönen, J., and Kaski, K. (2006). A model for social networks. Physica A, 371:851860.

Vázquez, A. (2003). Growing network with local rules: Preferential attachment, clustering hierarchy, and degree correlations. Physical Review E, 67:056104.

Wong, L. H., Pattison, P., and Robins, G. (2006). A spatial model for social networks. Physica A, 360(1):99-120.

zu Erbach-Schoenberg, E., Bullock, S., and Brailsford, S. (2014). A model of spatially constrained social network dynamics. Social Science Computer Review. 0894439313511934. 